\title{
An Unusual Treatment for Chronic Myelomonocytic Leukemia
}

\author{
Eric Warner, MSIII \\ Thomas Jefferson University, eric.warner@students.jefferson.edu
}

Neil Palmisiano, MD

Thomas Jefferson Univeristy, Neil.Palmisiano@jefferson.edu

Follow this and additional works at: https://jdc.jefferson.edu/tmf

Part of the Internal Medicine Commons

Let us know how access to this document benefits you

\section{Recommended Citation}

Warner, MSIII, Eric and Palmisiano, MD, Neil (2020) "An Unusual Treatment for Chronic Myelomonocytic Leukemia," The Medicine Forum: Vol. 21 , Article 6.

DOI: https://doi.org/10.29046/TMF.021.1.008

Available at: https://jdc.jefferson.edu/tmf/vol21/iss1/6

This Article is brought to you for free and open access by the Jefferson Digital Commons. The Jefferson Digital Commons is a service of Thomas Jefferson University's Center for Teaching and Learning (CTL). The Commons is a showcase for Jefferson books and journals, peer-reviewed scholarly publications, unique historical collections from the University archives, and teaching tools. The Jefferson Digital Commons allows researchers and interested readers anywhere in the world to learn about and keep up to date with Jefferson scholarship. This article has been accepted for inclusion in The Medicine Forum by an authorized administrator of the Jefferson Digital Commons. For more information, please contact: JeffersonDigitalCommons@jefferson.edu. 


\title{
An Unusual Treatment for Chronic Myelomonocytic Leukemia
}

\author{
Eric Warner, MSIII, Neil Palmisiano, MD
}

\begin{abstract}
Chronic myelomonocytic leukemia is a rare, aggressive, chronic leukemia that frequently progresses to acute myeloid leukemia. For younger patients, treatment ideally involves bone marrow transplant, and, if not a candidate, hydroxyurea or hypomethylating agents are the standard of care for symptomatic patients. Here, we present an unusual treatment of a patient with chronic myelomonocytic leukemia (CMML) characterized by atypical mutations in IDH2 and NPM-1 using Venetoclax and a hypomethylating agent with complete response.
\end{abstract}

\section{INTRODUCTION}

Chronic myelomonocytic leukemia has features of both myelodysplastic syndrome and myeloproliferative neoplasms. Characterized by an increase in circulating monocytes, the disease frequently progresses to acute myeloid leukemia (AML) and is the most aggressive chronic leukemia. Clonal cytogenetic abnormalities are found in approximately $30 \%$ of patients, most commonly trisomy 8 , trisomy 21 , monosomy 7 and del7q, and other complex karyotypes. ${ }^{1}$ For younger, fit patients, treatment ideally involves bone marrow transplant. If not a candidate, cytoreductive strategies using hydroxyurea or hypomethylating agents (HMA) are the standard of care for symptomatic patients. Because of the rarity of this disease, advancement of treatment strategies outside of transplant have been few. ${ }^{2}$ This case is presented to demonstrate an unusual treatment for CMML with IDH2 mutation using Venetoclax and an HMA.

\section{CASE PRESENTATION}

A 76-year-old female with a past medical history of an extraosseous chondrosarcoma status-post resection thirty years prior, hypertension, hyperlipidemia, and hypothyroidism presented to her primary care physician with fatigue, two weeks of dyspnea on exertion, lightheadedness, and nausea. The patient's primary care physician ordered a complete blood count, which was significant for a hemoglobin of $6.8 \mathrm{~g} / \mathrm{dl}$ and she was instructed to go to the Emergency Room for workup. In the ER, the patient was afebrile, and her vital signs were all within normal limits. On exam, she was noted to have a 3/6 systolic ejection murmur. Her lungs were clear to auscultation bilaterally and she did not have any skin abnormalities. She was given a transfusion of packed red blood cells and her hemoglobin responded appropriately. However, she was found to have thrombocytopenia and a leukocytosis with an absolute monocyte count of 5800 cells/uL ( $16 \%$ of the differential), a total white blood cell count of 36,400 cells/uL, a platelet count of 52,000 per/uL and no eosinophilia.

After being discharged, the patient was seen by oncology and underwent a bone marrow biopsy. Pathology showed hypercellular marrow (95\%) with trilineage proliferation, 10\% monocytes and 5-10\% blasts with less than $10 \%$ dysplasia. Flow cytometry, cytogenetics and Next-Generation Sequencing were unable to be performed due to an inadequate sample. Peripheral blood was then analyzed which showed $16 \%$ monocytes, $7 \%$ blasts and flow cytometry positive for CD4, CD11C, CD13, CD33, CD34, CD38, CD64, HLA-DR and CD117. Cytogenetics showed a normal female karyotype and was negative for BCR/ABL rearrangement. Mutational analysis was positive for IDH2, DNMT3A and NPM1 and was negative for JAK2, CALR and MPL. She was subsequently diagnosed with CMML, given her monocytic predominance and flow cytometry. Due to her excellent functional status (ECOG score of 1), curative treatment with cytoreductive agents and bone marrow transplant was pursued. Because of her NPM-1 and IDH2 mutation, it was decided that a regimen consisting of Decitabine and Venetoclax was appropriate. She began treatment in early February 2019 and, after two cycles, a biopsy was repeated, which showed only $9 \%$ abnormal myelocytes and no blasts. The regimen was well-tolerated by the patient and by June she was in complete response (CR) with no complications from treatment. She underwent another biopsy prior to transplant which showed hypocellular marrow with less than $1 \%$ monocytes and blasts. She then underwent non-myeloablative bone marrow transplant in November and, as of last follow-up 112 days later, is in recovery. Bone marrow biopsy post-transplant showed normocellular marrow with no increase in abnormal monocytes.

\section{DISCUSSION}

Chronic myelomonocytic leukemia is diagnosed based on a peripheral monocyte count above $10 \%$, in addition to bone marrow dysplasia. It frequently progresses to an acute leukemia in up to $20 \%$ of cases. ${ }^{3}$ The median age at onset is during the early seventh decade of life, and it has a slight male preference. There are several classification 


\section{The Medicine Forum, Vol. 21 [2020], Art. 6}

systems including the World Health Organization classification and the Mayo prognostic model, however the most commonly used is the CMML-specific prognostic scoring system (CPSS). The CPSS classifies patients into various risk categories using their CMML FAB type, cytogenetics, red blood cell transfusion dependence and, WHO subtyping based on the percentage of blasts in the periphery and bone marrow. For low risk patients the median survival is 6 years, for high risk patients it is just 5 months. ${ }^{1}$

The most common mutations in CMML have differing mechanisms, including epigenetic control of methylation (TET2 60\%), chromatin modulating (ASXL1 40\%), cell signaling pathways (RAS pathway 30\%) and mRNA splicing (SRSF2 50\%). ASXL1 has been shown to correlate with survival. Mutations in IDH1 or IDH2 are uncommon in CMML, occurring in less than $5 \%$ of patients. ${ }^{4}$ Although rare in $C M M L$, approximately $15 \%$ of patients with $A M L$ have a mutation in IDH. Normally IDH is involved in the citric acid cycle and metabolizes isocitrate to 5-alphaketoglutarate. When IDH is mutated, it gives rise to a new metabolic product: 2-hydroxyglutarate (2-HG). 2-HG accumulates and inhibits other enzymes including TET2 and JMJC, both epigenetic modifiers that can lead to leukemogenesis. ${ }^{5}$ Cells following this pathway become dependent on the anti-apoptotic gene Bcl-2 for survival. ${ }^{6}$

Due to its association with epigenetic dysregulation, the standard of care for CMML is a hypomethylating agent, specifically azacitidine or decitabine. These medications work by demethylating and subsequently allowing expression of hypermethylated tumor suppressor genes? Unfortunately, only $50 \%$ of patients respond to these agents, with less than $20 \%$ achieving complete response to these agents alone. ${ }^{1}$ HMAs are also frequently used in patients with AML who are not candidates for standard chemotherapy induction. Unfortunately, response rates in most studies of patients with AML show a complete response in only approximately $20 \% .{ }^{8}$ In more recent studies, patients over the age of 65 with AML were given the $\mathrm{Bcl}-2$ inhibitor Venetoclax in addition to an HMA. Twenty-four percent of these patients achieved CR, with an additional $43 \%$ achieving CR with incomplete cell count recovery. ${ }^{9}$ To our knowledge, there have been no studies using HMAs plus Venetoclax in patients with CMML, however, Venetoclax has been shown to have efficacy in CLL, AML, and ALL, particularly in patients with $\mathrm{IDH}$ mutations..$^{10}$ Although this is a relatively rare mutation in CMML, further study of this treatment regimen is warranted in patients who express IDH mutations in CMML and other MDS/MPNs.

\section{REFERENCES}

1. Patnaik, M. M., \& Tefferi, A. (2020). Chronic Myelomonocytic leukemia: 2020 update on diagnosis, risk stratification and management. American journal of hematology, 95(1), 97-115.

2. Zeidan, A. M., Hu, X., Long, J. B., Wang, R., Ma, X., Podoltsev, N. A., Huntington, S. F., Gore, S. D., \& Davidoff, A. J. (2017). Hypomethylating agent therapy use and survival in older patients with chronic myelomonocytic leukemia in the United States: A large population-based study. Cancer, 123(19), 3754-3762.

3. Arber, D. A., Orazi, A., Hasserjian, R., Thiele, J., Borowitz, M. J., Le Beau, M. M. Bloomfield, C. D., Cazzola, M., \& Vardiman, J. W. (2016). The 2016 revision to the World Health Organization classification of myeloid neoplasms and acute leukemia. Blood, 127(20), 2391-2405

4. Patnaik, M. M., \& Tefferi, A. (2016). Cytogenetic and molecular abnormalities in chronic myelomonocytic leukemia. Blood cancer journal, 6(2), e393.

5. Itzykson, R., Kosmider, O., Renneville, A., et al. (2013). Prognostic score including gene mutations in chronic myelomonocytic leukemia. Journal of clinical oncology, 31(19), 2428-2436.

6. Chan, S. M., Thomas, D., Corces-Zimmerman, M. R., Xavy, S., Rastogi, S., Hong, W. J., Zhao, F., Medeiros, B. C., Tyvoll, D. A., \& Majeti, R. (2015). Isocitrate dehydrogenase 1 and 2 mutations induce BCL-2 dependence in acute myeloid leukemia. Nature medicine, 21(2), 178-184

7. Datta, J., Ghoshal, K., Motiwala, T., \& Jacob, S. T. (2012). Novel Insights into the Molecular Mechanism of Action of DNA Hypomethylating Agents Role of Protein Kinase C delta in Decitabine-Induced Degradation of DNA Methyltransferase 1. Genes \& cancer, 3(1), 71-81.

8. Malik, P., \& Cashen, A. F. (2014), Decitabine in the treatment of acute myeloid leukemia in elderly patients. Cancer management and research, 6, 53-61

9. DiNardo, C. D., Pratz, K., Pullarkat, V., Jonas, B. A., Arellano, M., Becker, P. S., Frankfurt, O., Konopleva, M., Wei, A. H., Kantarjian, H. M., Xu, T., Hong, W. J., Chyla, B., Potluri, J., Pollyea, D. A., \& Letai, A. (2019). Venetoclax combined with decitabine or azacitidine in treatment-naive, elderly patients with acute myeloid leukemia. Blood., 133(1), 7-17.

10. Scheffold, A., Jebaraj, B. M. C., \& Stilgenbauer, S. (2018). Venetoclax: Targeting BCL2 in Hematological Cancers. Recent results in cancer research., 212, 215-242. 